\title{
Collocation Approximation to Eigenvalues of an Ordinary Differential Equation: Numerical Illustrations*
}

\author{
Carl de Boor and Blair Swartz
}

\begin{abstract}
We display the numerical results associated with the collocation of three eigenvalue problems using from one to four Gauss points per partition interval in order to document the sharpness of the error bounds we have previously obtained. The ordinary differential operators involved are real with constant coefficients; two of the problems have an eigenvalue whose ascent exceeds one. We propose an explanation for the observed manner in which a set of simple approximate eigenvalues can approach a single multiple eigenvalue.
\end{abstract}

Introduction. This is the second in a sequence of three papers concerned with the approximation of an eigenvalue of an $m$ th order ordinary differential equation $M x=\lambda N x$ (subject to $m$ homogeneous side conditions) through the use of piecewise polynomial projection methods as exemplified by collocation. We present here the numerical experiments which illustrate (indeed, pointed us toward) the convergence results we have previously described and proved [4]. The ascent, $\alpha$, of an ODE's eigenvalue plays a crucial role in the bounds obtained on the convergence rate of approximating eigenvalues from the known convergence rates associated with the nonsingular problem $M x=y$. For this reason, we first describe a technique which can sometimes be applied to determine this ascent a priori. We then pose eigenvalue problems for three constant coefficient operators: one problem in which $N$ is not the identity; others in which the order of the operator $M$, along with the eigenvalue's ascent and algebraic multiplicity, is two and three, respectively. Numerical results follow concerning the approximate eigenvalues associated with collocation at $k$ (from one to four) Gauss points per partition interval; most of them exemplify the sharpness of our $\theta\left(|\Delta|^{2 k / \alpha}\right)$ error estimates for each approximating eigenvalue and of our $\theta\left(|\Delta|^{2 k}\right)$ estimates for certain averages of all approximating eigenvalues. But, in one case involving a uniform partition, the individual numerical approximations of a multiple eigenvalue appear to converge faster than these estimates. We prove this is not illusory when collocating this problem with piecewise quadratics, concluding that the approximating eigenvalues

Received March 10, 1980.

1980 Mathematics Subject Classification. Primary 65L15, 65Jxx.

Key words and phrases. Eigenvalues, ordinary differential equation, collocation, piecewise polynomial, ascent of an eigenvalue, asymptotics of eigenvalue approximations.

*Sponsored by the United States Army under Contract No. DAAG29-75-C-0024 and by the United States Department of Energy under Contract No. W-7405-ENG.36.

By acceptance of this article, the publisher recognizes that the U. S. Government retains a nonexclusive, royalty-free license to publish or reproduce the published form of this contribution, or to allow others to do so, for U. S. Government purposes. 
then have the same ascent (two) as the approximated eigenvalue. In most of the cases concerning the approximation of a multiple eigenvalue, however, the numerically obtained eigenvalues appear to be simple and to converge to their common limit along equiangular rays in the complex plane. We end with an elementary explanation of this asymptotic phenomenon.

Continuing our first paper [4] on these matters, we begin with

5. A Way to Find the Ascent of an Eigenvalue of a Differential Equation. In this section we develop a technique which, in some circumstances, enables one to tell what the ascent of an eigenvalue of an ordinary differential equation actually is. The technique resembles one-parameter shooting; it is applied later to determine the ascent of an eigenvalue in each of the three problems we chose for our numerical examples.

As usual, we consider our eigenvalue problem

$$
\begin{gathered}
M x=\lambda N x \quad \text { on }(0,1), \\
M=D^{m}+\sum_{i<m} a_{i} D^{i}, \quad N=\sum_{i=0}^{n} b_{i} D^{i}, \quad n<m,
\end{gathered}
$$

(with sufficiently smooth coefficients) subject to the homogeneous side conditions (all of order less than $m$ )

$$
\begin{gathered}
\beta_{1} x=\cdots=\beta_{m-1} x=0, \\
\beta_{m} x=0,
\end{gathered}
$$

under which $M$ is presumed to have an inverse, $M^{-1}$. We suppose $\Lambda(\neq 0)$ is an eigenvalue. We also presume we have in hand another linear functional $\bar{\beta}_{m}$ which has the property that, for $\lambda$ near $\Lambda$, there is a unique solution $u_{\lambda}$ to the problem $M u_{\lambda}=\lambda N u_{\lambda}$ when $u_{\lambda}$ is subject to the $m-1$ side of conditions (1) together with the final inhomogeneous condition $\bar{\beta}_{m} u_{\lambda}=1$. The map

$$
\lambda \mapsto u_{\lambda}
$$

thereby defined is a smooth map for $\lambda$ near $\Lambda$ (take $u_{\lambda} \in C[0,1]$, say). (In particular, in each of our three examples, $M$ and $N$ are constant coefficient operators, the $m-1$ conditions (1) are the initial conditions $\beta_{i} x=\left(D^{i-1} x\right)(0), \bar{\beta}_{m} x$ is the initial condition $\left(D^{m-1} x\right)(0)$, and $\beta_{m} x$ is some linear combination of derivatives at the end points. The map $\lambda \mapsto u_{\lambda}$ is then analytic.)

In these general circumstances we have the following Facts:

I. $\left(\Lambda, u_{\Lambda}\right)$ is an eigenelement if and only if $F: \lambda \mapsto \beta_{m} u_{\lambda}$ has a zero at $\Lambda$. (In fact, the final Fact fixes the ascent of $\Lambda$ at the precise order of this zero.)

II. The eigenvalue $\Lambda$ is simple geometrically; i.e., its ascent equals its algebraic multiplicity. (For if $(\Lambda, y)$ is an eigenelement, then $x:=y-\left(\bar{\beta}_{m} y\right) u_{\Lambda} \in$ $\operatorname{ker}(M-\Lambda N)$ and satisfies (1) together with $\bar{\beta}_{m} x=0$; i.e., $x=0$.)

III. If $\lambda_{0}, \ldots, \lambda_{r}$ are near $\Lambda$, then

$$
\left(M-\lambda_{0} N\right)\left[\lambda_{0}, \ldots, \lambda_{r}\right] u_{\lambda}=\left[\lambda_{1}, \ldots, \lambda_{r}\right] N u_{\lambda},
$$

with $\left[\lambda_{i}, \ldots, \lambda_{j}\right] f_{\lambda}$ the $(j-i)$ th divided difference with respect to the parameter $\lambda$.

For

$$
\begin{gathered}
\left(M-\lambda_{0} N\right)\left[\lambda_{0}, \ldots, \lambda_{r}\right] u_{\lambda}=\left[\lambda_{0}, \ldots, \lambda_{r}\right]\left(\lambda N u_{\lambda}\right)-\lambda_{0}\left[\lambda_{0}, \ldots, \lambda_{r}\right] N u_{\lambda} \\
=1 \cdot\left[\lambda_{1}, \ldots, \lambda_{r}\right] N u_{\lambda}+\lambda_{0}\left[\lambda_{0}, \ldots, \lambda_{r}\right] N u_{\lambda}-\lambda_{0}\left[\lambda_{0}, \ldots, \lambda_{r}\right] N u_{\lambda}
\end{gathered}
$$


using Leibniz' formula; see, e.g., de Boor [1, p. 5]. Consequently,

IV. $u_{\lambda}^{[r]}:=\left(D_{\lambda}\right)^{r} u_{\lambda} / r$ ! satisfies not only the $m-1$ side conditions (1) but also the recursion

$$
(M-\lambda N) u_{\lambda}^{[r]}=N u_{\lambda}^{[r-1]}, \quad u_{\lambda}^{[0]}:=u_{\lambda} .
$$

V. If $F: \lambda \mapsto \beta_{m} u_{\lambda}$ has a zero of order at least $\alpha$ at $\Lambda$, then

(a) $\left(u_{\Lambda}^{[r]}\right)_{0}^{\alpha-1}$ satisfy the side conditions (1) and (2). (For $d^{r} F / d \lambda^{r}=\beta_{m} d^{r} u_{\lambda} / d \lambda^{r}$ $=r ! \beta_{m} u_{\lambda}^{[r]}$.)

(b) $\mu:=1 / \Lambda$ is an eigenvalue of $T:=N M^{-1} \cdot \mu$, too, is simple geometrically, and its ascent is the ascent of $\Lambda$.

(c) With

$$
v^{[r]}:=M u_{\Lambda}^{[r]}, \quad 0 \leqslant r<\alpha,
$$

we have $v^{[0]}=M u_{\Lambda} \in \operatorname{ker}(\mu-T)$, and (from (3))

$$
(\mu-T) v^{[r]}=\mu T v^{[r-1]}, \quad 1<r<\alpha .
$$

(d) $v^{[0]} \neq 0$, while, for $1 \leqslant r<\alpha, v^{[r]} \in \operatorname{span}\left(v^{[i]}\right)_{i<r}$ would imply

$$
\mu^{2 r} v^{[0]}=\mu^{r} T^{r} v^{[0]}=(\mu-T)^{r} v^{[r]} \in(\mu-T)^{r} \operatorname{span}\left(v^{[i]}\right)_{i<r}=\{0\} .
$$

Hence $v^{[0]}, \ldots, v^{[\alpha-1]}$ are linearly independent, and

$$
v^{[r]} \in \operatorname{ker}(\mu-T)^{r+1} \backslash \operatorname{ker}(\mu-T)^{r} .
$$

The same statements hold for the sequence $\left(T v^{[r]}\right)_{0}^{\alpha-1}$.

(e) The ascent of $\mu$ is at least $\alpha$. (For $v^{[\alpha-1]} \in \operatorname{ker}(\mu-T)^{\alpha} \backslash \operatorname{ker}(\mu-T)^{\alpha-1}$.)

VI. If $F: \lambda \mapsto \beta_{m} u_{\lambda}$ has a zero of exact order $\alpha$ at $\Lambda$, then the ascent of $\mu$ is $\alpha$; i.e., the ascent of $\Lambda$ is $\alpha$. For if the ascent of $\mu$ were to exceed $\alpha$, there would be some $f$ satisfying the side conditions (1), (2) so that

$$
(\mu-T) M f=\mu T v^{[\alpha-1]} .
$$

But then

$$
(M-\Lambda N) f=N u_{\Lambda}^{[\alpha-1]}=(M-\Lambda N) u_{\Lambda}^{[\alpha]}
$$

using (3). It would follow that $f-u_{\Lambda}^{[\alpha]} \in \operatorname{ker}(M-\Lambda N)$; therefore $f-u_{\Lambda}^{[\alpha]}=c u_{\Lambda}$ (since both satisfy the $m-1$ side conditions (1)). But then, as both $f$ and $u_{\Lambda}$ also satisfy condition (2), it would follow that

$$
0=\beta_{m} u_{\Lambda}^{[\alpha]}=\left.\left(d^{\alpha} F / d \lambda^{\alpha}\right)\right|_{\lambda=\Lambda} / r !
$$

and this would contradict the assumption that $\Lambda$ is a zero of $F$ of exact order $\alpha$.

6. Three Numerical Experiments: Problem Descriptions and Summary of Results. In this section we recall the numerical experiments which led us to conjecture the conclusions proved in the first three sections of this paper [4]. The experiments seemed necessary because previously published results, as noted in [4], proved more tantalizing than conclusive.

Three eigenvalue problems ((5.0)-(5.2)) were considered, all with constant coefficients and non-self-adjoint operators $M$. As mentioned in the previous section, the geometric multiplicity of all eigenvalues was one since $m-1$ side conditions were the usual conditions $\left(D^{i-1} x\right)(0)=0,1<i \leqslant m-1$. 
Problem I illustrates the case of an operator $N$ of order greater than zero. It uses the third order equation

$$
\begin{aligned}
L x & :=D^{3} x+a_{2} D^{2} x+a_{1} D x+a_{0} x=0, \quad t \text { on }(0,1), \\
a_{2} & :=-5, \quad a_{1}:=20, \quad a_{0}:=17.8111159551842
\end{aligned}
$$

under boundary conditions

$$
0=x(0)=(D x)(0)=x(1) .
$$

One solution takes the form

$$
x(t)=\exp (a t)-\exp (b t)[\cos (c t)+(a-b) \sin (c t) / c],
$$

where

$$
a=-0.73544611132431, \quad b=2.86772305566215, \quad c=3.99928443790975 .
$$

(These numbers were obtained as follows. If $x$ has the above general form with $a$ and $b$ given by

$$
\begin{aligned}
& b=b\left(c, a_{2}\right):=\left\{\left[a_{2}^{2}+3\left(c^{2}-a_{1}\right)\right]^{1 / 2}-a_{2}\right\} / 3, \\
& a=a\left(c, a_{2}\right):=-\left(a_{2}+2 b\right), \quad c \text { arbitrary }
\end{aligned}
$$

and with

$$
a_{1}=20, \quad a_{0}=a_{0}\left(c, a_{2}\right):=-a\left(b^{2}+c^{2}\right), \quad a_{2} \text { arbitrary, }
$$

then $L x=0$ and $x(0)=(D x)(0)=0$. Then, for $a_{2}=-5$, the function $f$ given by

$$
f\left(c, a_{2}\right):=x(1) /\left(D^{2} x\right)(0)
$$

has a zero, determined numerically as $c=3.99 \ldots$; the numbers $a, b, a_{0}$ associated with this choice for $c$ are exhibited above.)

We obtained Problem I by choosing

$$
M:=L-a_{2} D^{2}, \quad N=D^{2}
$$

so that an eigenvalue for (5.0)-(5.2) is $\lambda=5$. We became convinced that the ascent of this eigenvalue is one through the following exercise: having fixed the value of $a_{0}=a_{0}\left(c, a_{2}\right)$ at $17.811 \ldots$, the definitions of $a_{0}\left(c, a_{2}\right), b\left(c, a_{2}\right)$, and $a\left(c, c_{2}\right)$ determine $c$ as a function of $a_{2}$ in such a way that $x$ satisfies $L x=0$ and $x(0)=(D x)(0)=0$. Then (for $a_{2}$ near -5$)$

$$
F(\lambda)=F\left(-a_{2}\right):=f\left(c\left(a_{2}\right), a_{2}\right) \quad\left(=x(1) /\left(D^{2} x\right)(0)\right)
$$

is the function $F=\beta_{3} u_{\lambda}$ of Section 5. Numerically, we found $c(-5 \mp \varepsilon)$ and corresponding $F_{ \pm}:=F(5 \pm \varepsilon), \varepsilon=.05(-.01) .01$; we report the following difference quotients centered upon $\lambda=5$ :

$$
\begin{array}{cccccc}
\varepsilon & .05 & .04 & .03 & .02 & .01 \\
{\left[\left(F_{+}-F_{-}\right) / 2 \varepsilon-0.134260\right] \times 10^{6}} & 101 & 65 & 36 & 16 & 4 .
\end{array}
$$

We took this (apparent) $\varepsilon^{2}$ convergence as sufficient evidence that $d F /\left.d \lambda\right|_{\lambda=5} \neq 0$; i.e., that the ascent of this eigenvalue is one.

A description of the numerical method used and details of the numerical results are given in the next section. These results exhibit the kind of "superconvergence" one associates with collocating a third order nonsingular problem, both for the (real) approximate eigenvalue and for the breakpoint values of the approximate eigenfunction. 
The second and third problems were chosen to exhibit results for eigenvalues of ascent greater than one.

Problem II is a second order problem from Coddington and Levinson [5, p. 312]:

$$
M:=D^{2}, \quad N:=1,
$$

under boundary conditions

$$
0=x(0)=(D x)(0)+(D x)(1) .
$$

The functions required to determine the ascent as in Section 5 are

$$
u_{\lambda}(t)=\sin \sqrt{-\lambda} t / \sqrt{-\lambda}, \quad F(\lambda)=\beta_{2} u_{\lambda}=1+\cos \sqrt{-\lambda}
$$

so that $F$ vanishes if and only if $\lambda=-(K \pi)^{2}, K$ an odd integer. At each such eigenvalue $d F / d \lambda$ also vanishes while $d^{2} F / d \lambda^{2}=-1 /\left(4|\lambda|^{3 / 2}\right) \neq 0$. Thus the ascent of each eigenvalue is two. We fixed upon $\lambda=-\pi^{2}$; the eigenfunction and generalized eigenfunction are then, respectively,

$$
x_{1}(t)=\sin (\pi t), \quad x_{2}(t)=t \cos (\pi t)
$$

one notes that $(M-\lambda) x_{2}=-2 \pi x_{1}$ (as it should), while both satisfy the boundary conditions.

The numerical results for Problem II are amusing in that for uniform partitions one obtains unexpected "superconvergence", and only for a nonuniform partition (we took

$$
\left.t_{i}=\xi_{i} /\left(2-\xi_{i}\right), \quad \xi_{i}=i / l, \quad 0<i<l\right)
$$

did we observe the splitting of the approximate eigenvalues into two simple ones, converging as the square-root of the "superconvergent" rate from opposite (and not necessarily real) directions. Appendix I provides a proof, in the piecewise quadratic case, that the approximate eigenvalues associated with uniform partitions are real, have ascent two, and therefore converge as fast as their average; i.e., at the rate $\theta\left(|\Delta|^{2}\right)$.

Friedman [8, p. 226] presents a second order problem whose (complex) eigenvalue has ascent two under separated (complex) boundary conditions.

Problem III is a third order problem with ascent $(\lambda)=\alpha=3=\operatorname{alg} \cdot \operatorname{mult}(\lambda)$ :

$$
M:=D^{3}, \quad N:=1,
$$

under the separated boundary conditions

$$
0=x(0)=(D x)(0)=x(1)+b(D x)(1)+c\left(D^{2} x\right)(1) .
$$

The constants and eigenvalue $\lambda=r^{3}$, namely

$$
b=-0.11629166020407, \quad c=0.0094658373919757, \quad r=-10.205352107415 \text {, }
$$

were determined by shooting from $t=0$ (see Appendix II) so that the function $F(\lambda)=\beta_{3} u_{\lambda}$ of Section 5 had a zero of third order; i.e., the ascent of this eigenvalue is three. 
Approximations for this problem were considered only for the previously described nonuniform partition; the approximate eigenvalues appeared to be simple and to converge at essentially the same rate (the cube-root of the "superconvergent" rate) along three equiangular rays emanating from $\lambda$ in the complex plane. (The piecewise cubic case seems an exception, and, as it appears to contradict the Proposition in Section 8, we feel that we may not have gotten close enough to the asymptotic situation in that case.)

In Problems II and III, the geometric, harmonic, and arithmetic means of the $a$ approximate eigenvalues (also of their reciprocals) converged, as hoped, at the appropriate "superconvergent" rates.

Approximate eigenfunctions converging at this same rate at the breakpoints were easily constructed using these mean values and solving, by collocation, the appropriate approximating initial value problem. Such approximations are not necessarily eigenfunctions of any approximate operator, for they fail to satisfy the boundary condition at $t=1$ exactly. The eigenfunctions of the approximate operators in Problems II and III, themselves, cannot be expected to exhibit much accuracy. After all, an exact eigenfunction also satisfies a nonsingular problem $M x-\lambda N x=0$, under side conditions $\left(\beta_{i} x=0\right)_{1}^{m-1}$ together with an additional independent inhomogeneous side condition which we may regard as a normalization. Thus the corresponding eigenfunction of the approximate operator is also the collocation approximation to a nonsingular approximating problem $M y-$ $\lambda_{\Delta} N y=0$; it is very close to the solution of the latter problem and consequently only within $\theta\left(\left|\lambda-\lambda_{\Delta}\right|\right)$ of the solution of the former. This is borne out by the numerical experiments; and the fact that, as noted in Section 2 of [4], the invariant subspace is approximated (for $\alpha=3$ ) to within $\theta\left(|\Delta|^{k}\right.$ ) (which is better than $\theta\left(\left|\lambda-\lambda_{\Delta}\right|\right)=\theta\left(|\Delta|^{2 k / 3}\right)$ simply means that the error in each of these three approximations to the single eigenfunction lies mainly in their span.

7. Three Numerical Experiments: Method and Tabulated Results. Each of the three eigenvalue problems of Section 6 has the form

$$
M x=\lambda N x \text { in }(0,1), \quad \text { order } M=m, \text { constant coefficients, }
$$

subject to $m-1$ homogeneous initial conditions $\left(D^{i-1} x\right)(0)=0$ together with an additional homogeneous side condition $\beta_{m} x=0$. In each problem we focus on how well a single known eigenvalue $\lambda$ is approximated. Approximate eigenelements $\left(\lambda_{\Delta}, x_{\Delta}\right), x_{\Delta}$ in $\mathbf{P}_{m+k, \Delta}^{m}$, are sought using collocation at $k$ Gauss points in each of the $l$ intervals of the partition $\Delta$. The basis chosen for $\mathbf{P}_{m+k, \Delta}^{m}$ was a set of B-splines with appropriately chosen (multiple) knots; information about such matters (including many computer programs) can be found in de Boor [1]. The associated matrix eigenvalue problem, involving the B-spline coefficient vector $c$, takes the general form

$$
\Re c-\lambda_{\Delta} \Re c=0 \quad(\Re \neq 1 \text { even when } N=1),
$$

where the $k l \times(k l+m)$ matrices $\Re$ and $\Re$ have the special almost block-diagonal structure described in de Boor and Swartz [2] and illustrated, e.g., in de Boor and Swartz [3]. To this system we adjoin the $m$ homogeneous side conditions induced by $\left(\beta_{i} x_{\Delta}=0\right)_{1}^{m}$. 
As indicated in Sections 5 and 6, the geometric multiplicity of the eigenvalue of interest is one. Taken with the $m-1$ initial conditions, this fact allows us to compute the approximate eigenvalues by straightforward shooting for the complex zeros of the function $\left(\beta_{m} x_{\Delta}\right)\left(\lambda_{\Delta}\right)$, using $\lambda_{\Delta}$ as the shooting parameter, while holding $\bar{\beta}_{m} x_{\Delta}=\left(D^{m-1} x_{\Delta}\right)(0)$ fixed at one. Indeed, using one interpretation of Gauss-point collocation, we may concisely describe the whole process as finding approximate eigenvalues using shooting with an implicit Runge-Kutta scheme.

The (complex) roots of $\left(\beta_{m} x_{\Delta}\right)\left(\lambda_{\Delta}\right)$ near $\lambda$ were sought out numerically using Müller's method as implemented in the computer program found in Conte and de Boor [6, pp. 74-80]. This program searches for a prescribed number of complex roots, given initial guesses which we took uniformly as 1.1 $\lambda$. (A less confused version of the program description may be found in the book's third edition.) The convergence parameters used in the program were

$$
\mathrm{EP} 1=10^{-12}, \quad \mathrm{EP} 2=10^{-14} \text {, }
$$

together with a third parameter EP3 $=10^{-7}$ which took effect if the last change in the root was that small relative to the magnitude of $\lambda-\lambda_{\Delta}$ (all three exits were used, in fact, at one time or another). The range of the number of iterations taken, per root, was (excluding asterisked places in the tables to follow)

$\begin{array}{ccccc}\text { Problem } & \text { I } & \text { II(uniform mesh) } & \text { II(nonuniform mesh) } & \text { III } \\ \text { iterations } & 7-14 & 7-32 & 6-30 & 7-28\end{array}$

with the higher figures associated generally with problems of large dimension. For the reader who is curious concerning the results of requests for additional roots, we report the following: the eigenvalue of Problem II has ascent two and, for uniform partitions, the ascents of the approximate eigenvalues also appear to be two. A request for two roots in an analogous problem (uniform mesh) yielded two real, almost identical roots near the analog of $-\pi^{2}$; a request for four (nonuniform mesh) yielded two simple approximations near the analog of $-\pi^{2}$, two near $-9 \pi^{2}$ (the next nearest eigenvalue).

Given a candidate for $\lambda_{\Delta}$, the linear system corresponding to the initial value problem would have been solved most efficiently (computationally) by solving a succession of local linear systems, block by block, beginning with the $(k+m) \times$ $(k+m)$ block corresponding to the initial interval. Nevertheless, the method actually used was the complex band-matrix solver embedded in the LINPACK collection of subroutines [7]; for this our input specified and supplied $m+k-1$ super- and sub-diagonals constructed from the output of de Boor's B-spline package [1]. (These matrices were readily available, having been previously used in a relatively inefficient numerical method based, not on shooting, but on finding a zero of the determinant of the linear system (1) (plus side conditions) via Gaussian elimination.)

The tables to follow concern the convergence of the eigenvalues obtained numerically on a CDC 7600 using single-precision arithmetic. The errors recorded in the tables are the magnitude of the relative error. The "rates" recorded are the slopes of the relevant secant lines on a log-log plot of these errors against $1 / l$, where $l$ is the number of mesh intervals. In these tables, the number $-1.23-4$ represents $-1.23 \times 10^{-4}$. Asterisks follow errors presumed to be contaminated by 
roundoff problems or by problems associated with switches in convergence criteria for Müller's method. For complex $\lambda_{\Delta}$, we also report the coordinates of $\exp \left[i \operatorname{Arg}\left(\lambda_{\Delta}-\lambda\right)\right]$, i.e., the numbers

$$
\operatorname{RE}:=\text { Real part }\left(\lambda_{\Delta}-\lambda\right) /\left|\lambda_{\Delta}-\lambda\right|, \quad \text { IE }:=\text { Imag. part }\left(\lambda_{\Delta}-\lambda\right) /\left|\lambda_{\Delta}-\lambda\right| \text {. }
$$

In the cases of nonuniform meshes, the harmonic mean of the simple approximating $\lambda_{\Delta}$ 's (i.e., the reciprocal of the arithmetic mean of the corresponding $\mu_{\Delta}$ 's) was generally better than the arithmetic or geometric means-sometimes by an order of magnitude (but not always-e.g., in Problem II, nonuniform mesh, $k=2$, the error in the harmonic mean was up to three times that of the geometric mean, and in Problem III, $k=2$, the error in the harmonic was up to four times that of the arithmetic mean). For nonuniform meshes, then, we report the simple approximating eigenvalues (but not necessarily in the irregular order in which Müller's method found them) and immediately follow them with the data for their harmonic mean.

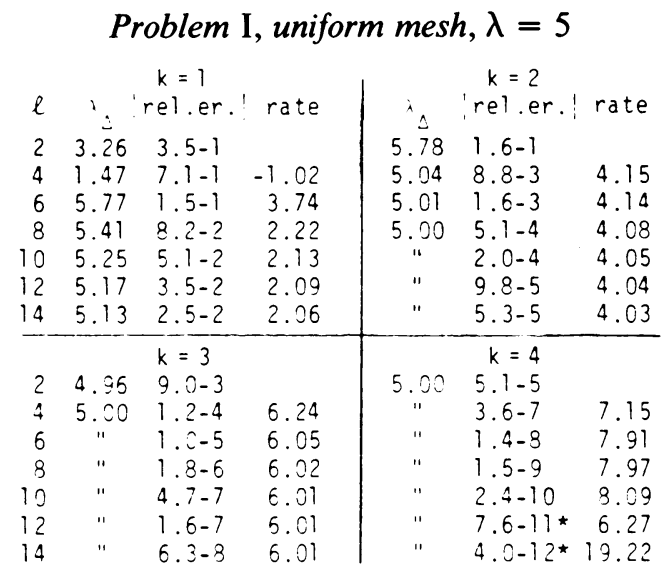

Otherwise, we note the following:

1. The hoped for superconvergent rates (modulo the ascent of the eigenvalue) are borne out by the results.

2. The real, simple eigenvalue in Problem I is approximated by a real eigenvalue; the direction of convergence depends on $k$.

3. The errors in the pair of simple eigenvalues approximating the real, multiple eigenvalue in Problem II (nonuniform mesh) are essentially real (imaginary) when $k$ is odd (even), and they have opposite sign.

4. Roundoff problems prevent the multiple approximate eigenvalue of Problem II (uniform mesh) from yielding better than $10^{-7}$ error; errors 100 times better could be attained (with more work, though) by the geometric mean of the two simple eigenvalues associated with the nonuniform mesh.

5. The error in one of the three simple eigenvalues in Problem III (nonuniform mesh) is always real; convergence to $\lambda$ takes place along three equiangular curves for $k>1$; for $k=4$, exhibited rates exceed provable rates.

6. The case $k=1$ of Problem III (nonuniform mesh) is curious, for the errors in two of the simple eigenvalues seem to approach zero at a rate $\theta\left(l^{-.65}\right)$ along curves whose slopes are almost \pm 1 in quadrants $I$ and IV, while the error in the third appears to be on the negative real axis and to have the faster rate (but larger magnitude) $\Theta\left(l^{-.74}\right)$. Were these rates in fact true asymptotically, it would contradict Proposition 8.1 (take $\beta=2$ in $(8.2 \mathrm{~b}$ ) in the next section) which asserts that 
the slower two must converge from opposite directions. We prefer to believe (and the table may bear this out) that the rates exhibited have not yet attained their asymptotic behavior.

Problem II, uniform mesh, $-\lambda=\pi^{2} \approx 9.87$

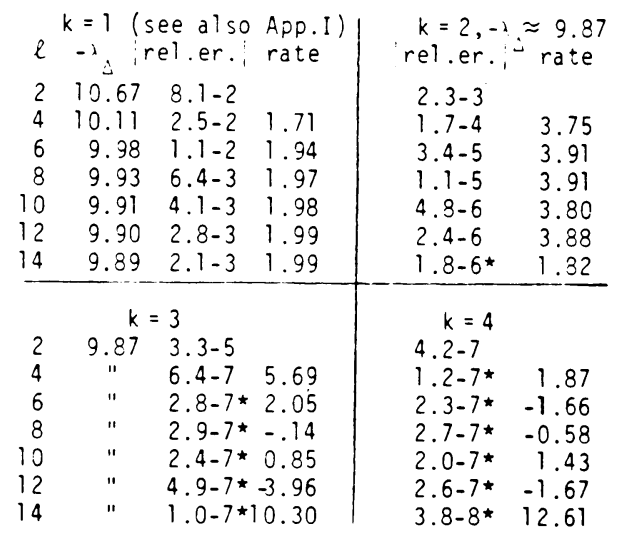

Problem II, nonuniform mesh (6.1), $-\lambda=\pi^{2} \approx 9.87$

Two simple approximating eigenvalues and their harmonic mean

\begin{tabular}{|c|c|c|c|c|c|c|c|c|}
\hline & \multicolumn{3}{|c|}{$\begin{array}{l}k=1, R E= \pm 1, I E=0 \\
-\operatorname{Re}\left(\lambda_{\Delta}\right) \text { |rel.er. | rate }\end{array}$} & \multicolumn{2}{|r|}{$\begin{array}{l}k=2, \\
\text { rel.er. }\end{array}$} & \multicolumn{3}{|c|}{$-\operatorname{Real}\left(\lambda_{\Lambda}\right) \approx 9.87$} \\
\hline 4 & $\begin{array}{r}13.51 \\
7.97 \\
10.03\end{array}$ & $\begin{array}{l}3.7-1 \\
1.9-1 \\
1.6-2\end{array}$ & & & $\begin{array}{l}4 \cdot 9-2 \\
4 \cdot 9-2 \\
2.2-4\end{array}$ & & $\begin{array}{l}-0.05 \\
-0.05 \\
-1.00\end{array}$ & $\begin{array}{r}1.00 \\
-1.00 \\
-0.00\end{array}$ \\
\hline 8 & $\begin{array}{r}11.26 \\
8.83 \\
9.90\end{array}$ & $\begin{array}{l}1.4-1 \\
1.1-1 \\
3.0-3\end{array}$ & $\begin{array}{l}1.39 \\
0.87 \\
2.42\end{array}$ & & $\begin{array}{l}1.2-2 \\
1.2-2 \\
3.4-5\end{array}$ & $\begin{array}{l}2.06 \\
2.06 \\
2.66\end{array}$ & $\begin{array}{r}-0.01 \\
-0.01 \\
1.00\end{array}$ & $\begin{array}{r}1.00 \\
-1.00 \\
-0.00\end{array}$ \\
\hline 12 & $\begin{array}{r}10.73 \\
9.16 \\
9.88\end{array}$ & $\begin{array}{l}8.7-2 \\
7.2-2 \\
1.3-3\end{array}$ & $\begin{array}{l}1.17 \\
0.92 \\
2.10\end{array}$ & & $\begin{array}{l}5.1-3 \\
5.1-3 \\
9.1-6\end{array}$ & $\begin{array}{l}2.05 \\
2.05 \\
3.27\end{array}$ & $\begin{array}{r}-0.00 \\
-0.00 \\
1.00\end{array}$ & $\begin{array}{r}1.00 \\
-1.00 \\
0.00\end{array}$ \\
\hline 16 & $\begin{array}{r}10.50 \\
9.33 \\
9.88\end{array}$ & $\begin{array}{l}6.4-2 \\
5.5-2 \\
7.1-4\end{array}$ & $\begin{array}{l}1.11 \\
0.94 \\
2.04\end{array}$ & & $\begin{array}{l}2.8-3 \\
2.8-3 \\
3.1-6\end{array}$ & $\begin{array}{l}2.03 \\
2.03 \\
3.70\end{array}$ & $\begin{array}{r}-0.00 \\
-0.00 \\
1.00\end{array}$ & $\begin{array}{r}1.00 \\
-1.00 \\
-0.00\end{array}$ \\
\hline 20 & $\begin{array}{r}10.36 \\
9.43 \\
9.87 \\
\end{array}$ & $\begin{array}{l}5 \cdot 0-2 \\
4 \cdot 5-2 \\
4.5-4\end{array}$ & $\begin{array}{l}1.08 \\
0.95 \\
2.02 \\
\end{array}$ & & $\begin{array}{l}1.8-3 \\
1.8-3 \\
1.3-6 \\
\end{array}$ & $\begin{array}{l}2.02 \\
2.02 \\
3.83\end{array}$ & $\begin{array}{r}-0.00 \\
-0.00 \\
1.00\end{array}$ & $\begin{array}{r}1.00 \\
-1.00 \\
-0.00\end{array}$ \\
\hline 4 & $\begin{array}{c}k=3, R \\
9.93 \\
9.82 \\
9.87\end{array}$ & $\begin{aligned}= & \pm 1, \text { IE }= \\
& 5.6-3 \\
& 5.5-3 \\
& 2.9-5\end{aligned}$ & & 4 & $\begin{array}{l}\quad k=4, \\
4.5-4 \\
4.5-4 \\
2.5-7\end{array}$ & & $\begin{array}{l}\left(\lambda_{\Delta}\right) \approx 0 \\
-0.00 \\
-0.00 \\
-1.00\end{array}$ & $\begin{array}{r}.87 \\
1.00 \\
-1.00 \\
-0.00\end{array}$ \\
\hline 8 & $\begin{array}{l}9.88 \\
9.86 \\
9.87\end{array}$ & $\begin{array}{l}6.8-4 \\
6.8-4 \\
2.9-7\end{array}$ & $\begin{array}{l}3.03 \\
3.01 \\
6.61\end{array}$ & 6 & $\begin{array}{l}9.4-5 \\
9.4-5 \\
8.4-9\end{array}$ & $\begin{array}{l}3.88 \\
3.88 \\
8.35\end{array}$ & $\begin{array}{l}-0.00 \\
-0.00 \\
-1.00\end{array}$ & $\begin{array}{r}1.00 \\
-1.00 \\
-0.01\end{array}$ \\
\hline 12 & " & $\begin{array}{l}2.0-4 \\
2.0-4 \\
2.1-8\end{array}$ & $\begin{array}{l}3.07 \\
3.06 \\
6.52\end{array}$ & 8 & $\begin{array}{l}2.9-5 \\
2.9-5 \\
8.7-10 *\end{array}$ & $\begin{array}{l}4.04 \\
4.04 \\
7.88\end{array}$ & $\begin{array}{l}-0.00 \\
-0.00 \\
-0.73\end{array}$ & $\begin{array}{r}1.00 \\
-1.00 \\
0.68\end{array}$ \\
\hline 16 & " & $\begin{array}{l}8.2-5 \\
8.2-5 \\
3.1-9\end{array}$ & $\begin{array}{l}3.04 \\
3.04 \\
6.68\end{array}$ & 10 & $\begin{array}{l}1.2-5^{\star} \\
1.2-5^{\star} \\
3.4-9^{\star}\end{array}$ & $\begin{array}{r}4.06 \\
4.06 \\
-6.10\end{array}$ & $\begin{array}{l}-0.00 \\
-0.00 \\
-0.01\end{array}$ & $\begin{array}{r}1.00 \\
-1.00 \\
1.00\end{array}$ \\
\hline 20 & " & $\begin{array}{l}4.2-5 \\
4.2-5 \\
9.6-10 \star\end{array}$ & $\begin{array}{l}3.03 \\
3.03 \\
5.16\end{array}$ & 12 & $\begin{array}{l}5.7-6^{\star} \\
5.7-6^{\star} \\
1.5-9^{\star}\end{array}$ & $\begin{array}{l}3.97 \\
3.97 \\
4.37\end{array}$ & $\begin{array}{r}-0.00 \\
0.00 \\
0.96\end{array}$ & $\begin{array}{r}1.00 \\
-1.00 \\
0.29\end{array}$ \\
\hline
\end{tabular}

The second author undertakes to preserve other data (including errors in approximating eigenfunctions obtained by the initial value technique previously mentioned) for the next decade and to reproduce them (upon reasonable request) during that time. 
Problem III, nonuniform mesh (6.1), $-\lambda \approx 1063$

Three simple approximating eigenvalues and their harmonic mean

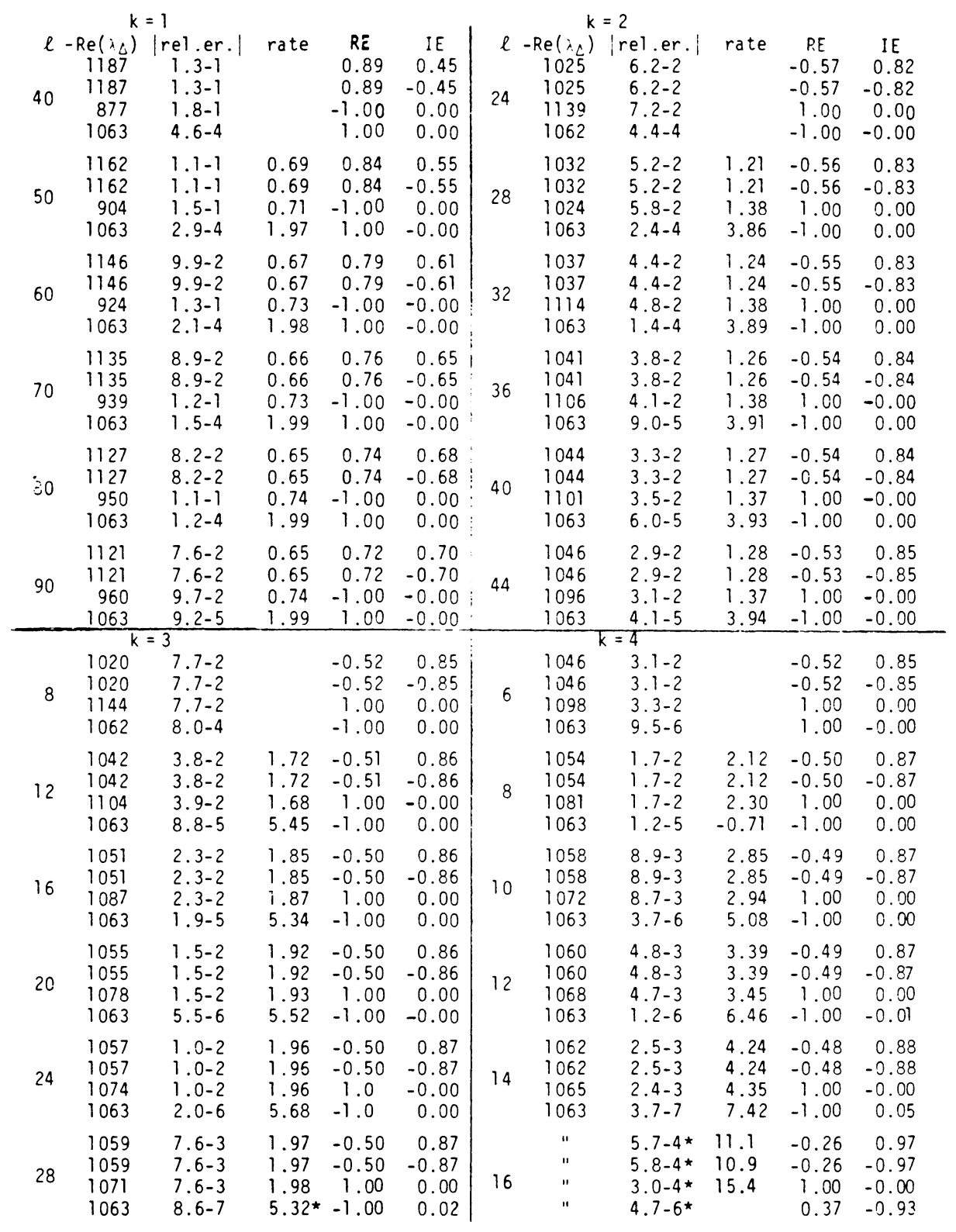

8. An Observation About a Multiple Eigenvalue's Asymptotics. Two of our numerical examples concern problems in which the algebraic multiplicity, $a$, coincides with the ascent, $\alpha$, where both are greater than one. In these examples the approximate eigenvalues (for a sequence of nonuniform partitions) were observed to be simple and to converge to their common limit along equiangular rays in the complex plane. In this section we offer an elementary explanation of this phenomenon. 
The conclusions concerning the approximate solution of eigenvalue problems which are relevant here are the following (justification for them is found in Part I of this paper [4]). One is given (in principle) an $a \times a$ matrix $J$ of complex numbers whose sole complex eigenvalue $\mu \neq 0$ has algebraic multiplicity $a$ (of course) and ascent $\alpha$; we suppose $\alpha>1$. One is similarly given a sequence of $a \times a$ matrices $\left(J_{n}\right)_{1}^{\infty}$; they arise from the collocation process using a sequence $\left(\Delta_{n}\right)_{1}^{\infty}$ of partitions of $[0,1]$ whose maximum interval lengths satisfy $\left|\Delta_{n}\right| \rightarrow 0$. The $a$ eigenvalues of $J_{n}$ are denoted by $\left(\mu_{j}^{(n)}\right)_{1}^{a}$. Their reciprocals are the approximate eigenvalues one actually calculates in collocation, the ones which approximate the single desired eigenvalue $1 / \mu$. One knows that $J_{n} \rightarrow J$ in the following sense: there is a sequence $\left(\delta_{n}\right)_{1}^{\infty}$ of positive numbers converging to zero, computable in principle from the partition sequence, so that

$$
J-J_{n}=\theta\left(\delta_{n}\right) .
$$

Consequently each $\mu_{j}^{(n)}$ approximates $\mu$ to the extent that

$$
\mu_{j}^{(n)}=\mu+\theta\left(\left(\delta_{n}\right)^{1 / \alpha}\right), \quad 1 \leqslant j \leqslant a .
$$

(A sequence of matrices $\left(Z^{(n)}\right)_{1}^{\infty}$ and a sequence of complex numbers $\left(w_{n}\right)_{1}^{\infty}$ satisfy the order relation $Z^{(n)}=\theta\left(w_{n}\right)$ if and only if there is some $K>0$ and some integer $N$ so that $\max _{i, j}\left|Z_{i j}^{(n)}\right| \leqslant K\left|w_{n}\right|, n \geqslant N$. They satisfy $Z^{(n)}=o\left(w_{n}\right)$ if and only if for every $\varepsilon>0$ there is some $N(\varepsilon)$ so that $\max _{i, j}\left|Z_{i j}^{(n)}\right|<\varepsilon\left|w_{n}\right|$ for $n>N(\varepsilon)$. $Z^{(n)}=o(1)$ if and only if $Z^{(n)} \rightarrow 0$.)

We now suppose there are $r$ "worst" approximate eigenvalues. By this we mean that, for each $n,\left(\mu_{j}^{(n)}\right)_{1}^{a}$ can be and has been reordered so that

$$
K^{-1} \leqslant\left|\mu_{i}^{(n)}-\mu\right| /\left|\mu_{1}^{(n)}-\mu\right| \leqslant K
$$

eventually for $i=1, \ldots, r$, while $\mu_{i}^{(n)}-\mu=o\left(\mu_{1}^{(n)}-\mu\right), i>r$. Indeed, we shall assume more: that the error in each has the beginnings of an asymptotic expansion in that of the first. More precisely, we suppose that, for each $n,\left(\mu_{j}^{(n)}\right)_{1}^{a}$ can be and has been reordered to form a vector $\boldsymbol{\mu}^{(n)} \in \mathbf{C}^{a}$ which satisfies

$$
\boldsymbol{\mu}^{(n)}=\boldsymbol{\mu}+\varepsilon_{n}[\mathbf{w}+o(1)]
$$

with

$$
\varepsilon_{n}:=\mu_{1}^{(n)}-\mu \rightarrow 0, \quad \mu:=(\mu, \ldots, \mu)^{T} \in \mathbf{C}^{a},
$$

and $\mathbf{w}$ some fixed complex $a$-vector. (In such circumstances $w_{1}$ is necessarily one, and the number of worst eigenvalues is the number of nonzero components of $\mathbf{w}$.)

In addition, we assume that the $r$ worst approximate eigenvalues converge slower than the known bound $\delta_{n}$ on $J-J_{n}$, i.e.,

$$
\delta_{n}^{1 /(\beta-1)}=o\left(\varepsilon_{n}\right) \text { for some integer } \beta \text { with } 2 \leqslant \beta \leqslant \alpha .
$$

Very loosely speaking, $\mu^{(n)}$ is a differentiable perturbation of $\boldsymbol{\mu}$ with respect to the error in $\mu_{1}^{(n)}$, and the error in $\mu_{1}^{(n)}$ is sufficiently bad relative to the known bounds on $J-J_{n}$.

A situation such as we have just described could be anticipated for collocation if a sequence of partitions $\left(\Delta_{n}\right)_{1}^{\infty}$ of $[0,1]$ was constructed by specifying each partition 
point in $\Delta_{n}$ using $\left(t_{i}^{(n)}=f(i / n)\right)_{i=0}^{n}$, where $f$ is some sufficiently smooth homeomorphism of $[0,1]$. In this case, if $k$ collocation points are specified for each partition interval using $k$ fixed reference points $\left(\rho_{i}\right)_{1}^{k}$ in $[-1,1]$ (independent of $n$ and of $\Delta_{n}$ ), then a known sequence for the bound (1) on $J-J_{n}$ is given by $\delta_{n}=\left|\Delta_{n}\right|^{k}$. If the $k$ reference points are the $k$ Gauss points, then one may instead take $\delta_{n}=\left|\Delta_{n}\right|^{2 k}$. We offer no justification for (2) other than that it appears to be satisfied (with $\beta=\alpha$ ) in some of our numerical examples.

Finally, for $\mathbf{v} \in \mathbf{C}^{a}$, let

$\sigma_{j}(\mathbf{v}):=$ the $j$ th elementary symmetric function of the components of $\mathbf{v}$; for example, $\sigma_{1}(v)=v_{1}+\cdots+v_{a}$; the geometric mean of $\left(v_{j}\right)_{1}^{a}$ is $\sigma_{a}(v)^{1 / a}$; the harmonic mean, $a \sigma_{a}(\mathbf{v}) / \sigma_{a-1}(v)$. Then we offer simple proof of the following

Proposition 1. Suppose $J-J_{n}$ is bounded as in (1), while the sequence of vectors $\left(\mu^{(n)}\right)_{1}^{\infty}$ of approximate eigenvalues satisfies (2). Then there are at least $\beta$ worst approximate eigenvalues; i.e., $\left(w_{j} \neq 0\right)_{1}^{\beta}$. Furthermore, $\left(\sigma_{j}(w)=0\right)_{1}^{\beta-1}$. Finally, if there are exactly $\beta$ worst approximate eigenvalues, i.e., if $\left(w_{j}=0\right)_{\beta+1}^{a}$, then there is some complex number const $\neq 0$ such that

$$
w_{j}=\text { const } \exp (2 \pi i j / \beta), \quad 1 \leqslant j \leqslant \beta .
$$

The proof begins with a fact concerning the elementary symmetric functions.

LemMA 2. For any vector $\mathbf{v} \in \mathbf{C}^{a}$ and with $\mu=(\mu, \ldots, \mu)^{T} \in \mathbf{C}^{a}$,

$$
\sigma_{j}(\mu+\mathbf{v})=\sum_{i=0}^{j} \sigma_{i}(\mathbf{v})\left(\begin{array}{c}
a-i \\
j-i
\end{array}\right) \mu^{j-i}, \quad 0 \leqslant j \leqslant a .
$$

Proof (for completeness' sake). Express each side of the equation

$$
\prod_{j=1}^{a}\left[z-\left(\mu+v_{j}\right)\right]=\prod_{j=1}^{a}\left[(z-\mu)-v_{j}\right]
$$

in terms of the elementary symmetric functions

$$
\sum_{j=0}^{a}(-1)^{j} \sigma_{j}(\mu+\mathbf{v}) z^{a-j}=\sum_{j=0}^{a}(-1)^{j} \sigma_{j}(\mathbf{v})(z-\mu)^{a-j} .
$$

Now equate coefficients of corresponding powers of $z$.

Up to a sign, the characteristic polynomials of the $a \times a$ matrices $J_{n}$ are

$$
p_{n}(z)=z^{a}+q_{n}(z), \quad\left(\operatorname{degree}\left(q_{n}\right)=a-1\right),
$$

and they constitute a sequence of perturbations of the characteristic polynomial $(z-\mu)^{a}=z^{a}+q(z)$ of the $a \times a$ matrix $J$. The next result says (roughly) that if, for such polynomials, the perturbed zeros have a certain asymptotic form and if the slowest converging zero converges slower than the $p$ th root of the rate at which the $p$ highest order coefficients of $q_{n}$ are converging (to those of $q$ ), then at least $p+1$ perturbed zeros converge this slowly. The proof is a simple extension of the obvious proof for the case $p=1$. 
LEMMA 3. Let $\left(\mathbf{v}_{n}\right)_{1}^{\infty}$ be a sequence of vectors in $\mathbf{C}^{a}$ of the form

$$
\mathbf{v}_{n}=\mathbf{w}+o(1) \quad\left(\text { some } \mathbf{w} \in \mathrm{C}^{a} \text { independent of } n\right)
$$

which satisfies, for some sequence $\left(\varepsilon_{n}\right)_{1}^{\infty}$ of complex numbers converging to zero, for some complex number $\mu$, and for some positive integer $p$, the relations

$$
\sigma_{j}\left(\mu+\varepsilon_{n} \mathbf{v}_{n}\right)=\sigma_{j}(\mu)+o\left(\left(\varepsilon_{n}\right)^{p}\right), \quad 1 \leqslant j \leqslant p,
$$

where $\boldsymbol{\mu}:=(\mu, \ldots, \mu)^{T} \in \mathbf{C}^{a}$. Then $\sigma_{j}(\mathbf{w})=0,1<j<p$. Consequently, the components of $\mathbf{w}$ consist of the a zeros of the polynomial

$$
\pi(z):=z^{a}+\sum_{j=p+1}^{a}(-1)^{j} \sigma_{j}(\mathbf{w}) z^{a-j}
$$

so that if $a-p$ or more components of $\mathbf{w}$ are zero, then $\mathbf{w}=\mathbf{0}$.

Proof. We suppress the subscript $n$. Because of Lemma 2, and since $\sigma_{0}(\varepsilon v)=1$ while $\sigma_{j}(\mu)=\left(\begin{array}{l}a \\ j\end{array}\right) \mu^{j}$, assumption (4) means that

$$
\sigma_{j}(\mu)+\sum_{i=1}^{j} \sigma_{i}(\varepsilon \mathbf{v})\left(\begin{array}{c}
a-i \\
j-i
\end{array}\right) \mu^{j-i}=\sigma_{j}(\mu)+o\left(\varepsilon^{p}\right), \quad 1 \leqslant j \leqslant p .
$$

Consequently, the vector $\sigma:=\left(\sigma_{j}(\varepsilon v)\right)_{1}^{p} \in C^{p}$ satisfies the linear system $\mathcal{L} \sigma=o\left(\varepsilon^{p}\right)$, where $\mathcal{L}$ is a lower triangular matrix, independent of $n$, whose main diagonal consists entirely of ones. Hence $\|\sigma\|_{\infty}=o\left(\varepsilon^{p}\right)$. Now, $\sigma_{j}(\varepsilon v)=\varepsilon^{j} \sigma_{j}(v)$, so that

$$
\sigma_{j}(\mathbf{v})=o\left(\varepsilon^{p-j}\right)=o(1), \quad 1<j<p .
$$

But, from assumption (3),

$$
\sigma_{j}(\mathbf{v})=\sigma_{j}(\mathbf{w})+o(1), \quad 1 \leqslant j \leqslant p .
$$

Hence, $\sigma_{j}(w)=0(1 \leqslant j \leqslant p)$ since $\sigma_{j}(w)$ is independent of $n$ yet $o(1)$. Consequently,

$$
\prod_{j=1}^{a}\left(z-w_{j}\right)=z^{a}+\sum_{j=p+1}^{a}(-1)^{j} \sigma_{j}(\mathbf{w}) z^{a-j}
$$

Recall, now, how $\sigma_{j}(\mathbf{w})$ is a sum of products of $j$ components of $\mathbf{w}$.

Proof of Proposition 1. Using (1) (as remarked at (2.15) of Part I of this paper [4])

$$
\sigma_{j}\left(\mu^{(n)}\right)=\sigma_{j}(\mu)+\vartheta\left(\delta_{n}\right), \quad 1 \leqslant j \leqslant a .
$$

In Lemma 3, take $p:=\beta-1$. Then, with $\varepsilon_{n}:=\mu_{1}^{(n)}-\mu$,

$$
\sigma_{j}\left(\mu^{(n)}\right)=\sigma_{j}(\mu)+o\left(\left(\varepsilon_{n}\right)^{p}\right), \quad 1<j<p,
$$

by assumption (2b); furthermore, $\varepsilon_{n} \rightarrow 0$. We also take

$$
\mathbf{v}_{n}:=\left(\mu^{(n)}-\mu\right) / \varepsilon_{n}
$$

(unless $\varepsilon_{n}=0$ when we let $\mathbf{v}_{n}:=w$ given in (2a)). Because of assumption (2a) the hypotheses of Lemma 3 are now satisfied; we conclude that

$$
\sigma_{j}(\mathbf{w})=0, \quad 1<j<\beta .
$$

Furthermore, if more than $a-\beta$ components of $\mathbf{w}$ were zero, then $\mathbf{w}$ would be $\mathbf{0}$. But $w_{1}=1$, hence at least $\beta$ components of $w$ are not zero. Finally, if $\left(w_{j} \neq 0\right)_{1}^{\beta}$ but $\left(w_{j}=0\right)_{\beta+1}^{a}$, then

$$
\sigma_{j}(\mathbf{w})=0, \quad \beta<j \leqslant a,
$$


as well. Hence $\mathbf{w}$ consists of the $a$ roots of

$\pi(z)=z^{a}+\sum_{i=\beta}^{a}(-1)^{i} \sigma_{i}(\mathbf{w}) z^{a-i}=z^{a-\beta}\left(z^{\beta}-c\right), \quad c:=(-1)^{\beta+1} \prod_{1}^{\beta} w_{j} \neq 0$.

Contextual Remarks. The elementary results represented by Lemma 3 and Proposition 1 join a large body of work concerning the zeros of perturbed polynomials and the eigenvalues of perturbed matrices. Thus, as an immediate consequence of Lemma 3 we have the following result concerning differentiable perturbations of the polynomial $(z-\mu)^{a}$ :

COROllary 4. Suppose that, as the positive real variable $\chi$ discretely (if not continuously) approaches zero, the coefficients of the polynomial

$$
\sum_{i=0}^{a}(-1)^{i} \sigma_{i}(\mu(\chi)) z^{a-i} \quad\left(=\prod_{j=1}^{a}\left[z-\mu_{j}(\chi)\right]\right)
$$

take on the limiting values $\left(\begin{array}{c}a \\ i\end{array}\right)(-\mu)^{i}$, the leading $p+1$ coefficients approaching their limits with better than first order accuracy, i.e.,

$$
\left(d \sigma_{i} / d \chi\right)\left(0_{+}\right)=0, \quad 0 \leqslant i \leqslant p, \text { some } p>0 .
$$

Suppose, too, that for some $r \geqslant 1 / p, d \mu_{j} / d\left(\chi^{r}\right)$ exists at $\chi=0_{+}, 1 \leqslant j<a$. Then

$$
\sigma_{i}\left(\left[d \mu / d\left(\chi^{1 / p}\right)\right]\left(0_{+}\right)\right)=0, \quad 0<i<p,
$$

so that, not only does $\mu_{j} \rightarrow \mu, 1 \leqslant j \leqslant a$, but also at least $p+1$ components of $\left[d \mu / d\left(\chi^{1 / p}\right)\right]\left(0_{+}\right)$are not zero if a single component is not zero.

This may be compared, for example, with a stronger structure theorem of Wilkinson [10, pp. 64-65] which, under its stronger hypotheses and adapted to perturbations of $(z-\mu)^{a}$, says

THEOREM (WILKINSON). Let $\mu_{1}(\chi), \ldots, \mu_{a}(\chi)$ be the a zeros of a polynomial

$$
z^{a}+\sum_{i=1}^{a}(-1)^{i}\left[\left(\begin{array}{c}
a \\
i
\end{array}\right) \mu^{i}+p_{i}(\chi)\right] z^{a-i}
$$

here each $p_{i}$ is a polynomial which vanishes at $\chi=0$. Then $\left(\mu_{i}\right)_{1}^{a}$ consists of $b$ disjoint subsequences; the $a_{j}$ zeros in the $j$ th subsequence are the $a_{j}$ values of a series $\mu+c_{j} w+d_{j} w^{2}+\ldots$ corresponding to the $a_{j}$ different values of $w=\chi^{1 / a_{j}}$.

Wilkinson's result is observed in its eigenvalue context by Kato [9, footnote, $\mathrm{p}$. 73], who considers eigenvalue problems for both analytic matrices [9, Chapter II, Sections 1 through 4] and merely differentiable ones [op cit., Section 5]. (Kato's results become relevant if $\left(J_{n}\right)_{1}^{\infty}$ is somehow construed as a perturbation of $J$ via a single small continuous parameter $\chi$; in such circumstances the eigenvalues of $J(\chi)$ would constitute a " $\mu$-group".) For both kinds of perturbations, the eigenvalues are of course continuous; in the analytic case they can have only algebraic singularities. In both cases, the spectral projector associated with $J(\chi)$ is at least differentiable, although the individual spectral projectors associated with distinct approximate eigenvalues can have algebraic poles (or worse behavior) unless the ascent of $\mu$ is one. In both cases, if the ascent of $\mu$ is one, the approximate eigenvalues are shown differentiable (among a variety of other results). But when the ascent exceeds one and $J(\chi)$ is merely differentiable (a case somewhat analogous to the situation above), Kato offers little else. 
Appendix I: Quadratic Spline Eigenvalues Can Have Ascent Two if the Partition is Uniform; Convergence Can be $\mathcal{\theta}\left(h^{2}\right)$; Other Tridiagonal Schemes Can Have These Two Properties. Consider the (non-self-adjoint) eigenvalue problem from Coddington and Levinson [5, p. 312]

$$
D^{2} w-\lambda w=0, \quad w(0)=0, \quad D w(1)+D w(0)=0 .
$$

The eigenvalues are

$$
\lambda=-(k \pi)^{2}, \quad k \text { odd }, k>0 .
$$

From Sections 5 and 6 we conclude they have geometric multiplicity one and ascent two (hence their algebraic multiplicity, too, is two). The corresponding eigenfunctions are

$$
w_{1}(t)=\sin (k \pi t)
$$

while the corresponding generalized eigenfunctions

$$
w_{2}(t)=t \cos (k \pi t)
$$

satisfy the boundary conditions and (as they should)

$$
\left(D^{2}-\lambda\right) w_{2}=-2 k \pi w_{1} \text {, hence }\left(D^{2}-\lambda\right)^{2} w_{2}=0 .
$$

We consider the approximating problem obtained by collocating (1) with $C^{1}$ quadratic splines midway between a uniformly placed set of knots on $[0,1]$. We show that, with $h:=|\Delta|$,

(a) its eigenvalues are $\theta\left(h^{2}\right)$ accurate and have ascent two (except the most negative eigenvalue has ascent one if $l$ is odd),

(b) its eigenfunctions are, up to a constant factor, exact at the knots. If they are normalized by matching $D w_{1}(0)$ then they are $\theta\left(h^{2}\right)$ accurate at the knots. Either is $\vartheta\left(h^{2}\right)$ accurate in $\mathbf{L}_{\infty}$, as is its derivative,

(c) the corresponding generalized eigenfunctions are, with their first derivatives, $\vartheta\left(h^{2}\right)$ accurate in $\mathbf{L}_{\infty}$.

Thus, the collocation of (1) with $C^{1}$ quadratics on a uniform partition does not demonstrate the sharpness of the poor convergence rates we prove for problems whose eigenvalues have ascent $>1$. However, the collocation of (1) using a nonuniform partition can exhibit such poor convergence rates, as is seen in the numerical results for Problem II.

To prove (a)-(c) we begin by considering the second centered divided difference operator $D_{+} D_{-}$. Let $h=1 / l$. Then, if $u(t):=\exp [i K \pi(t-\varphi)](\varphi, K$ real $)$,

$$
\left(D_{+} D_{-}-\sigma\right) u=0, \quad \sigma:=-2(1-\cos \theta) / h^{2}, \quad \theta:=K \pi h .
$$

Let $v(t):=t u(t)$. Then

$$
D_{+} D_{-} v=\left(t D_{+} D_{-}+2 D_{0}\right) u, \quad D_{0}:=\left(D_{+}+D_{-}\right) / 2
$$

so that

$$
\left(D_{+} D_{-}-\sigma\right) v=i \nu u, \quad \nu:=2 \sin \theta / h .
$$

Letting $U(t):=\sin [K \pi(t-\varphi)]$, the imaginary part of (2) yields $\left(D_{+} D_{-}-\sigma\right) U$ $=0$. Setting $V(t):=(t-\varphi) \cos [K \pi(t-\varphi)]$, we have, from the real parts of (2) and (3), that

$$
\left(D_{+} D_{-}-\sigma\right) V=-\nu U, \text { hence }\left(D_{+} D_{-}-\sigma\right)^{2} V=0
$$


Let $c$ be the $(l+2)$-vector of coefficients of the $l+2$ translates of the quadratic B-spline associated with knots uniformly placed $h$ apart on $[0,1]$. Then the difference equations associated with collocating $D^{2}-\lambda$, using this basis, at the $l$ midpoints of a uniform mesh, are

$$
\left(D_{+} D_{-}-\lambda A\right) c=0, \quad A:=1+h^{2} D_{+} D_{-} / 8, \quad c=\left[\left(c_{j}\right)_{0}^{l+1}\right]^{T} .
$$

Let

$$
\bar{\sigma}:=\sigma /\left(1+\sigma h^{2} / 8\right) \quad \text { (the denominator is never zero). }
$$

Were the $j$ th coefficient given by $U\left(t_{j}\right)\left(V\left(t_{j}\right)\right.$, respectively), we would have

$$
\begin{aligned}
& \left(D_{+} D_{-}-\bar{\sigma} A\right) U=0, \\
& \left(D_{+} D_{-}-\bar{\sigma} A\right) V=-\nu\left(1-h^{2} \bar{\sigma} / 8\right) U,
\end{aligned}
$$

so that $\left(D_{+} D_{-}-\bar{\sigma} A\right)^{2} V=0$. Now, $K$ and $\varphi$ are completely arbitrary here; it is the boundary conditions which will determine them. These conditions, expressed in terms of the B-spline coefficients, are

$$
c_{0}+c_{1}=0, \quad\left(D_{+} c\right)_{0}+\left(D_{-} c\right)_{l+1}=0 .
$$

Pick, now,

$$
K=1,3, \ldots, 2\lfloor(l-1) / 2\rfloor+1=: K_{\max } ; \varphi:=h / 2,
$$

thereby determining B-spline coefficients (using $U$ and $V$, respectively)

$$
\begin{aligned}
(C)_{j}=\sin \left[K \pi\left(t_{j}-h / 2\right)\right], \quad t_{j}:=j h, & j=0, \ldots, l+1, K<K_{\max }, \\
(B)_{j}=\left(t_{j}-h / 2\right) \cos \left[K \pi\left(t_{j}-h / 2\right)\right], & K<K_{\max }(l \text { even }), K<K_{\max }(l \text { odd }) .
\end{aligned}
$$

The coefficient vector $C$ satisfies the boundary conditions (6); in view of (5) it is an eigenvector with associated eigenvalue $\bar{\sigma}=\bar{\sigma}_{K}$. The ascent of $\bar{\sigma}$ will be at least two, by (5), if the boundary conditions (6) are also satisfied by the coefficient vector $B$ of (8) with the same choice (7) for $K$. But with this choice it may be verified that

$$
\begin{aligned}
(B)_{0} & =-(B)_{1}=-h \cos (K \pi h / 2) / 2, \\
\left(D_{+} B\right)_{0} & =-\left(D_{-} B\right)_{l+1}=\cos (K \pi h / 2) .
\end{aligned}
$$

Hence the eigenvalues $\bar{\sigma}$ indeed have ascent at least two.

Since the algebraic multiplicity of $\lambda=-(K \pi)^{2}$ is two, there are exactly two approximate eigenvalues which converge to it; from (7), (4), and (2), we see that, eventually, both of these are $\bar{\sigma}_{K}$. Hence the algebraic multiplicity of $\bar{\sigma}_{K}$ is, eventually, at most two, so its ascent, as it exceeds one, is eventually exactly two. Because the average of the two eigenvalues approximating $\lambda$ converges at the superconvergent rate associated with collocation at the single Gauss point per partition interval, we conclude

$$
\overline{\boldsymbol{\sigma}}_{K}=\lambda+\mathcal{O}\left(h^{2}\right),
$$

the convergence constant being computable from (4) and (2).

Remark. In the same fashion, any sequence of approximate eigenvalues, all with ascent exceeding one, which converges to $\lambda$ (a sequence, say, associated with collocation of (1) at $k$ Gauss points per interval) will converge at the superconvergent rate $\theta\left(h^{2 k}\right)$. Unfortunately, such a sequence has not been found to enable us to analytically verify the numerical results for Problem II, uniform mesh, when $k$ exceeds one. 
$\Theta\left(h^{2}\right)$ superconvergence (to a given $\lambda$ of ascent and multiplicity two) of some sequence of approximate eigenvalues also would follow if the ascent of every approximate eigenvalue exceeds one. And, indeed, the ascent and algebraic multiplicity of the eigenvalues $\bar{\sigma}_{K}\left(K\right.$ specified by (7)) is exactly two (except for $\bar{\sigma}_{K_{\max }}$ if $l$ is odd) if one can show that the eigenvalues are distinct so that the $l$ vectors (8) span the $l$-space of coefficients which satisfy the two homogeneous constraints (6). But, from (2) and (4), $d \bar{\sigma} / d K<0$ for $0<K \pi h=\theta<\pi$. Consequently, since $\bar{\sigma}(K=0)=0,0>\bar{\sigma}_{K_{1}}>\bar{\sigma}_{K_{2}}$ if $0<K_{1} h<K_{2} h \leqslant 1$. Thus, the choice (7) indeed yields (for $h$ fixed) a strictly monotone decreasing sequence of negative eigenvalues whose ascents and algebraic multiplicities are all two, except, possibly, for the last.

Although approximate eigenfunctions are not of major concern in this paper, they are almost in hand for this example, and we pause now to consider them.

The value of the spline corresponding to $C$ of $(8)$, at a knot $t_{j}=j h$, is

$$
s_{C}\left(t_{j}\right)=\left(C_{j}+C_{j+1}\right) / 2=\cos (K \pi h / 2) \sin \left(\pi K t_{j}\right) .
$$

Hence, the spline eigenfunctions are exact at the knots-up to a constant factor. The mid-knot value of $s_{C}\left(\right.$ at $\left.\bar{t}_{j}:=\left(t_{j-1}+t_{j}\right) / 2\right)$ is

$$
s_{C}\left(\bar{t}_{j}\right)=\left[\left(1+h^{2} D_{+} D_{-} / 8\right) C\right]_{j}=\left(1+\sigma h^{2} / 8\right) C_{j}=w_{1}\left(\bar{t}_{j}\right)+\mathcal{O}\left(h^{2}\right) .
$$

Hence $\left\|s_{C}-w_{1}\right\|_{\infty}=\theta\left(h^{2}\right)$. If the spline eigenfunction is normalized by requiring that its derivative at $0,\left(D_{+} C\right)_{0}$, match that of $w_{1}$, then

$$
\begin{aligned}
s_{\text {norm }}\left(t_{j}\right) & =\{\pi K h /[2 \tan (\pi K h / 2)]\} \sin \left(\pi K t_{j}\right) \\
& =w_{1}\left(t_{j}\right)+\vartheta\left(h^{2}\right) ;
\end{aligned}
$$

the mid-knot values of $s_{\text {norm }}$ are also $\theta\left(h^{2}\right)$ accurate.

That the spline, $s_{B}$, corresponding to $B$ of (8), differs from the generalized eigenfunction $w_{2}(t)=t \cos (K \pi t)$ by $\mathcal{O}\left(h^{2}\right)$ may be seen as follows: $s_{B}(0)=w_{2}(0)$ while $s_{B}(1)=-\cos (K \pi h / 2)=w_{2}(1)+\vartheta\left(h^{2}\right)$. At the $j$ th mid-knot $\bar{t}_{j}$,

$$
\begin{aligned}
s_{B}\left(\bar{t}_{j}\right) & =\left[\left(1+h^{2} D_{+} D_{-} / 8\right) B\right]_{j} \\
& =\left[B+h^{2}(\sigma B-\nu C) / 8\right]_{j}=\bar{t}_{j} \cos \left(K \pi \bar{t}_{j}\right)+\vartheta\left(h^{2}\right) .
\end{aligned}
$$

Hence $\left\|s_{B}-w_{2}\right\|_{\infty}=\vartheta\left(h^{2}\right)$.

The derivatives of $s_{C}, s_{\text {norm }}$, and $s_{B}$ are piecewise linear. As each can be shown to be $\Theta\left(h^{2}\right)$ accurate at the knots, these derivatives are also $\Theta\left(h^{2}\right)$ accurate in $\mathbf{L}_{\infty}$.

As an aside, this Appendix also shows that the usual finite difference operator $D_{+} D_{-}$has eigenvalues and eigenfunctions with similar properties if the unknowns represent function values at the mid-knots $\left(\bar{t}_{i}\right)_{1}^{l}$ rather than B-spline coefficients; the boundary conditions (6) describe the usual extrapolations in this context. For the approximate eigenvalues for this problem are $\sigma_{K}$ (rather than $\bar{\sigma}_{K}$ ); the mesh point values of the eigenfunctions and generalized eigenfunctions $\left(\right.$ at $\left.\left(\bar{t}_{i}\right)_{1}^{l+1}\right)$ are given by (8). If we use this difference-scheme interpretation but take $A$ (above (4)) to be $1+h^{2} D_{+} D_{-} / 12$, then the approximate eigenvalues are

$$
\hat{\sigma}_{K}=\sigma_{K} /\left(1+\sigma_{K} h^{2} / 12\right)=\lambda_{K}+\vartheta\left(h^{4}\right) ;
$$


the difference scheme is then the Numerov scheme. The "linear spline Galerkin" scheme (along with the knot-collocated $C^{2}$ cubic spline scheme) has an $A=1+$ $h^{2} D_{+} D_{-} / 6$ and consequent $\theta\left(h^{2}\right)$ eigenvalue approximations (here we take $\left(\bar{t}_{j}\right)_{1}^{l}$ as knots and B-spline coefficients as unknowns; the collocated boundary conditions are satisfied by (8)).

Appendix II: An Eigenvalue Problem With Ascent Three. Consider the initial value problem

$$
\begin{gathered}
D^{3} u-\lambda u=0, \quad \lambda=r^{3}, r \neq 0 \\
u(0)=D u(0)=0, \quad D^{2} u(0)=3 r^{2} .
\end{gathered}
$$

Then $u(t)=f(r t)$, where

$$
f(y):=\exp (y)-2 \exp (-y / 2) \cos (\sigma y-\pi / 3), \quad \sigma:=\sqrt{3} / 2 .
$$

Furthermore,

$$
\left(D_{t} u\right)(t)=r g(r t), \quad\left(D_{t}^{2} u\right)(t)=r^{2} h(r t)
$$

where

$$
\begin{aligned}
& g(y):=\exp (y)-2 \exp (-y / 2) \cos (\sigma y+\pi / 3) \\
& h(y):=\exp (y)-2 \exp (-y / 2) \cos (\sigma y-\pi) .
\end{aligned}
$$

We seek numbers $a=1, b, c$ and a value $R$ such that the function

$$
G(r):=a u(1)+b\left(D_{t} u\right)(1)+c\left(D_{t}^{2} u\right)(1)
$$

has a zero of third order at $r=R \neq 0$. (The third homogeneous boundary condition for the eigenvalue problem will be taken to be $\left[a x+b D x+c D^{2} x\right](1)=$ 0 ; an eigenfunction will be $f(R t)$.) Forming $G^{\prime}$ and $G^{\prime \prime}$, we seek values $y=R$ such that the matrix

$$
\mathfrak{R}(y):=\left[\begin{array}{ccc}
f & y g & y^{2} h \\
f^{\prime} & (y g)^{\prime} & \left(y^{2} h\right)^{\prime} \\
f^{\prime \prime} & (y g)^{\prime \prime} & \left(y^{2} h\right)^{\prime \prime}
\end{array}\right]
$$

is singular. Using the relations $f^{\prime}=g, g^{\prime}=h, h^{\prime}=f$, we see that

$$
\Re(y)=\operatorname{diag}\left(1, y^{-1}, y^{-2}\right) \Re(y) \text {, }
$$

where the symmetric matrix $\Re$ is given by

$$
\Re(y):=\left[\begin{array}{ccc}
f & y g & y^{2} h \\
y g & y g+y^{2} h & 2 y^{2} h+y^{3} f \\
y^{2} h & 2 y^{2} h+y^{3} f & 2 y^{2} h+4 y^{3} f+y^{4} g
\end{array}\right] .
$$

Finally, then, we search for a zero $y=R$ of $\operatorname{det}[\Re(y)]$. The coefficients of $u(1)$, $(D u)(1)$, and $\left(D^{2} u\right)(1)$ are then the solution of $\mathscr{U}(R)(1, b, c)^{T}=0$. These constants are displayed in Section 6, Problem III.

That $R$ is a zero of $G$ of exact order three follows if

$$
G^{\prime \prime \prime}=f^{\prime \prime \prime}+b(y g)^{\prime \prime \prime}+c\left(y^{2} h\right)^{\prime \prime \prime} \neq 0 \text { at } y=R \text {. }
$$

This last is so since, as may be checked,

$$
G^{\prime \prime \prime}(R) / 3=b f(R)+2 c[R g(R)+f(R)] \neq 0 .
$$


Finally, we note that the functions required in Section 5 are

$$
\begin{gathered}
u_{\lambda}(t)=u(t) / 3 r^{2}=f\left(\lambda^{1 / 3} t\right) /\left(3|\lambda|^{2 / 3}\right), \quad\left(\lambda=r^{3}\right), \\
F(\lambda)=\beta_{3} u_{\lambda}=G\left(\lambda^{1 / 3}\right) /\left(3|\lambda|^{2 / 3}\right) .
\end{gathered}
$$

We observe that if $G$ has a zero of exact order three at $R \neq 0$, then $F$ also has such a zero at $\lambda=R^{3}$, since then

$$
d^{3} F /\left.d \lambda^{3}\right|_{\lambda=R^{3}}=G^{\prime \prime \prime}(R) /\left(3 R^{2}\right)^{4} \neq 0 .
$$

Mathematics Research Center

University of Wisconsin-Madison

610 Walnut Street

Madison, Wisconsin 53706

Theoretical Division

University of California

Los Alamos Scientific Laboratory

Los Alamos, New Mexico 87545

1. C. DE Boor, A Practical Guide to Splines, Appl. Math. Sci. Series \#27, Springer-Verlag, Berlin and New York, 1978.

2. C. DE Boor \& B. SwartZ, “Collocation at Gaussian points," SIAM J. Numer. Anal., v. 10, 1973, pp. 582-606.

3. C. DE BOOR \& B. SWARTZ, "Comments on the comparison of global methods for two-point boundary value problems," Math. Comp., v. 31, 1977, pp. 916-921.

4. C. DE BOOR \& B. SWARTZ, "Collocation approximation to eigenvalues of an ordinary differential equation: The principle of the thing," Math. Comp., v. 35, 1980, pp. 679-694.

5. E. A. Coddington \& N. Levinson, Theory of Ordinary Differential Equations, McGraw-Hill, New York, 1955.

6. S. D. Conte \& C. DE Boor, Elementary Numerical Analysis, 2nd ed., McGraw-Hill, New York, 1972.

7. J. J. Dongarra, C. B. Moler, J. R. Bunch \& G. W. Stewart, LINPACK Users' Guide, SIAM, Philadelphia, Pa., 1979.

8. B. Friedman, Principles and Techniques of Applied Mathematics, Wiley, New York, 1956.

9. T. Kato, Perturbation Theory for Linear Operators, Springer-Verlag, Berlin and New York, 1966.

10. J. H. Wilkinson, The Algebraic Eigenvalue Problem, Clarendon Press, Oxford, 1965. 